\title{
RECONCILIAÇÃO DADOS EM PROCESSAMENTO DE MINÉRIO UTILIZANDO O SIMULADOR EMSO
}

\author{
D. Q. F. MENEZES ${ }^{1}$, A. L., SAMPAIO, F. C. PEIXOTO ${ }^{1}$ e D. M. PRATA ${ }^{1}$ \\ ${ }^{1}$ Universidade Federal Fluminense, Departamento de Engenharia Química e de Petróleo. \\ E-mail para contato: pratadiego@gmail.com
}

\begin{abstract}
RESUMO - Este trabalho apresenta um estudo sobre reconciliação de dados em estado estacionário em processamento de minério, com base em uma unidade industrial publicada por Alhaj-Dibo et al. (2008). É realizada uma breve revisão bibliográfica sobre reconciliação de dados e sua aplicação em processamento de minério, basicamente: cominuição, flotação e hidrometalurgia. O problema estudado é constituído por 48 variáveis sendo 3 não medidas e 7 corrompidas por erros grosseiros, que invalidam a base estatística comumente adotada. O problema é resolvido no pacote computacional EMSO (Ambiente para Modelagem, Simulação e Otimização) que dispõe de rotinas computacionais propriamente desenvolvidas para reconciliação de dados em estado estacionário com restrições lineares ou não lineares. Resultados satisfatórios são obtidos e o desempenho do simulador EMSO pode ser comprovado, incluindo estimadores robustos para detecção dos múltiplos erros grosseiros.
\end{abstract}

\section{INTRODUÇÃO}

A reconciliação de dados (RD) representa uma etapa crucial para estratégias de controle e otimização em tempo real de processos industriais. Os processos de produção de um mineral ou planta metalúrgica têm como meta operar em condições ótimas, com base em índices de desempenho. Estes podem ser expressos por fatores técnicos, tais como a quantidade de material valioso produzido, ou pela qualidade do material produzido (por exemplo, pureza do metal). Por meio do procedimento de RD medidas de processo como vazões, concentrações e temperaturas são ajustadas de tal forma a satisfazer restrições, como balanços de massa e energia. Desta forma, a RD tem sido muito utilizada em processamento de minérios.

Neste procedimento, tradicionalmente, é considerado que essas medidas estão sujeitas apenas a erros pequenos, aleatórios e com distribuição Normal, o que resulta no estimador de Mínimos Quadrados Ponderados (MQP). Desta forma, uma reconciliação eficiente requer a eliminação ou compensação de outro tipo de erros não aleatórios, os erros grosseiros que, ao não satisfazer a hipótese de normalidade, invalidam o resultado. Estes erros podem se originar em falhas, má-calibração ou deterioração de sensores e flutuações súbitas de força. Um número considerável de abordagens foi proposto para a detecção de erro grosseiro (DEG). Quando mais de um erro grosseiro está presente é necessária uma estratégia (seqüencial ou simultânea) para detectá-los, e isto é conhecido como detecção de múltiplos erros grosseiros (DMEG). A literatura técnica tem mostrado que a utilização de distribuições de probabilidade oriundas da estatística robusta permite realizar a RD e DMEG simultaneamente, sem necessidade de eliminação dos erros grosseiros ou estratégias computacionalmente intensivas. 
O simulador EMSO (Soares e Secchi, 2003) dispõe de rotinas propriamente desenvolvidas para RD em estado estacionário com restrições lineares ou não lineares, incluindo testes tradicionais e estimadores robustos para realizar o procedimento de DMEG.

Seguindo esta direção, este trabalho apresenta um estudo sobre RD e DMEG em estado estacionário em processamento de minério, com base na unidade industrial publicada por Alhaj-Dibo et al. (2008), utilizando-se o simulado EMSO e o estimador robusto Lorenziana.

Este trabalho está organizado em cinco seções, além desta introdução. Na Seção 2 é realizada a revisão da literatura, ressaltando aplicações de $\mathrm{RD}$ em processamento de minério. A Seção 3 descreve a formulação do problema de reconciliação robusta de dados. Na Seção 4 é apresentado o problema estudado e o critério de avaliação. Na Seção 5 são apresentados os resultados. E, finalmente, na Seção 6 as conclusões do trabalho são apresentadas.

\section{REVISÃO DA LITERATURA}

Conjuntos de dados reais medidos intrinsecamente contêm erros, e desta forma não é de se esperar que obedeçam às leis de conservação como balanços de massa e energia. Portanto, um procedimento de retificação de dados é essencial para obter uma recuperação satisfatória da informação contida nos dados, para sua utilização em diferentes aplicações.

O procedimento mais utilizado na retificação é a $\mathrm{RD}$, onde dados medidos são ajustados de maneira estatisticamente coerente pelo estimador (função objetivo) resultante da formulação de máxima verossimilhança sobre a distribuição estatística dos erros de medição assumida, de forma a satisfazer às leis de conservação e demais restrições impostas (modelo matemático), obtendo estimativas confiáveis para as variáveis e parâmetros do processo. Tradicionalmente é assumida distribuição Normal, que resulta no estimador de MQP.

Para que esta finalidade seja atingida, dois outros procedimentos são geralmente usados durante o processo de retificação: a classificação das variáveis e a detecção de erros grosseiros. O primeiro determina se a informação disponível é suficiente para resolver o problema de reconciliação. O segundo procura por valores medidos que não seguem a distribuição estatística admitida, uma vez que estes a invalidam. Erros grosseiros dividem-se basicamente em: valores espúrios ("outliers") e desvios sistemáticos ("bias").

Prata et al. (2008) aponta três abordagens principais para realizar o procedimento de RD e DMEG simultaneamente: a análise de agrupamentos (clusters), as redes neuronais artificiais e os estimadores robustos, as quais podem ser utilizadas independentemente das restrições (estacionária ou dinâmica; linear ou não linear) e evitam eliminações de medidas com erros.

Os estimadores robustos têm sido muito reportados na literatura técnica para lidar com o problema de RD e DMEG simultaneamente - Reconciliação Robusta de Dados (RRD) -. Tjoa e Biegler (1991) provaram que usando o estimador baseado na distribuição Normal Contaminada, ao invés do tradicional MQP, qualquer valore espúrio presente nas medidas podia ser substituído por valores reconciliados, sem necessitar de esquemas iterativos. Prata $e t$ al. (2008) apresentaram um estudo comparativo entre alguns estimadores robustos em um reator químico. Os autores concluiriam que os estimadores Welsch e Lorenziana obtiveram os melhores resultados, comprovando o enorme potencial destes estimadores. 


\subsection{Reconciliação de Dados em Processamento de Minério}

RD é um procedimento comprovado para lidar com os erros de medição, estimar os valores das variáveis medidas e não medidas, e assim, melhorar a confiabilidade dos índices de desempenho calculados de processo. Estes são aspectos importantes para a indústria de processamento metalúrgico e de minerais, já que, em muitos casos, as variáveis estratégicas são medidas com precisão limitada, ou simplesmente não são medidas devido a custos proibitivos (Sbarbaro e DelVillar, 2010).

Parece que Weigel (1972) foi o pioneir a endereçar estudos de RD em processamento de minério, operando em estado estacionário, especificamente para um sistema de flotação $\mathrm{Cu}$ Zn. Hodouin et al. (1988) aplicaram RD em sistemas de flotação $\mathrm{Cu}-\mathrm{Zn}$ contendo metais preciosos. Outras aplicações de RD em de estado estacionário em processamento de minério, tais como trituração (Hodouin et al., 1981), moagem (Mular et al., 1976; White et al., 1977), extração de ouro (de Andrade, 2006), hidrometalurgia (Bazin et al., 2005), pirometalurgia (Eksteen et al., 2002; Bazin et al., 1998; Bazin et al., 2003), e preparação de cimento/clinquer (Hodouin et al., 1982) também foram reportadas. RD e DMEG simultânea também foram realizadas em processamento de minério. Du et al. (1997) utilizaram redes neuronais artificiais e Alhaj-Dibo et al. (2008) utilizaram estimador robusto. Sbarbaro e DelVillar (2010) apresentaram uma revisão completa e recente sobre RD em processamento de minério.

\section{O PROBLEMA DE RECONCILIAÇÃO ROBUSTA DE DADOS (RRD)}

Existem muitas classes de estimadores robustos, sendo as mais populares aquelas utilizadas nos estimadores-M, que são generalizações de um estimador de máxima verossimilhança (Prata et al., 2008). Assumindo que os erros de medição não são correlacionados, o problema de RRD estacionário, de forma generalizada, adota a formulação,

$$
\min \sum_{i} \rho\left(\frac{x_{i}-z_{i}}{\sigma_{i}}\right)=\min \sum_{i} \rho\left(\xi_{i}\right)
$$

sujeito a

$$
\begin{aligned}
& h[x, u]=\mathbf{0} \\
& g[x, u] \geq \mathbf{0}
\end{aligned}
$$

onde $\rho$ é uma função razoavelmente monotônica, $\xi_{i}$ e $\sigma_{i}$ são, respectivamente, o resíduo padronizado e o desvio padrão da variável discreta medida $z_{\mathrm{i}}, x$ e $u$ são os vetores das variáveis medidas reconciliadas e não medidas (observáveis) estimadas, respectivamente. Finalmente, $h$ e $g$ são as restrições algébricas de igualdade e desigualdade, respectivamente.

Apresentam-se os estimadores de MQP e Lorenziana como possíveis escolhas para $\rho$.

$$
\text { MQP: } \quad \rho_{M Q P}\left(\xi_{i}\right)=\frac{\xi_{i}^{2}}{2}
$$


Lorenziana: $\rho_{L}\left(\xi_{i}, c_{L}\right)=-\frac{1}{1+\left(\frac{\xi_{i}^{2}}{2 c_{L Z}^{2}}\right)}$

$\mathrm{Na}$ Equação $4 \mathrm{c}_{\mathrm{L}}$ é um parâmetro de sintonia relacionado à eficiência relativa, sendo calculada em relação a uma distribuição de referência, quase sempre adotada a distribuição Normal (Prata et al., 2008). Para uma eficiência relativa de $95 \%$ o valor de $c_{L}$ é 2,678.

Métodos usados para mensurar a robustez de um estimador envolvem a chamada função de influência (FI). Para os estimadores-M, a FI corresponde à derivada da função $\rho$ em relação ao resíduo padronizado $\left(\xi_{i}\right)$ e, de maneira simplificada, corresponde ao peso (influência) dado ao efeito da magnitude de um erro grosseiro (mensurado em termos de múltiplos do resíduo padronizado) sobre as estimativas. O estimador MQP não é robusto, pois sua função de influência é $\mathrm{FI}_{\mathrm{MQP}}=\xi_{\mathrm{i}}$, ou seja, a influência de erros grosseiros nas estimativas é ilimitada (falta de robustez) e aumenta proporcionalmente com o aumento da magnitude do erro grosseiro. Pelo contrário, o estimador Lorenziana possui FI que decresce de forma limitada tendendo à zero, que anula o efeito negativo de erros grosseiros sobre as estimativas, mesmo com o aumento de sua magnitude, por isso é classificado como "redescending". Já o estimador de Fair sofre influência dos erros grosseiros, mesmo que de forma limitada, por isso é classificado como "monótono". Esta comparação é ilustrada na Figura 1.

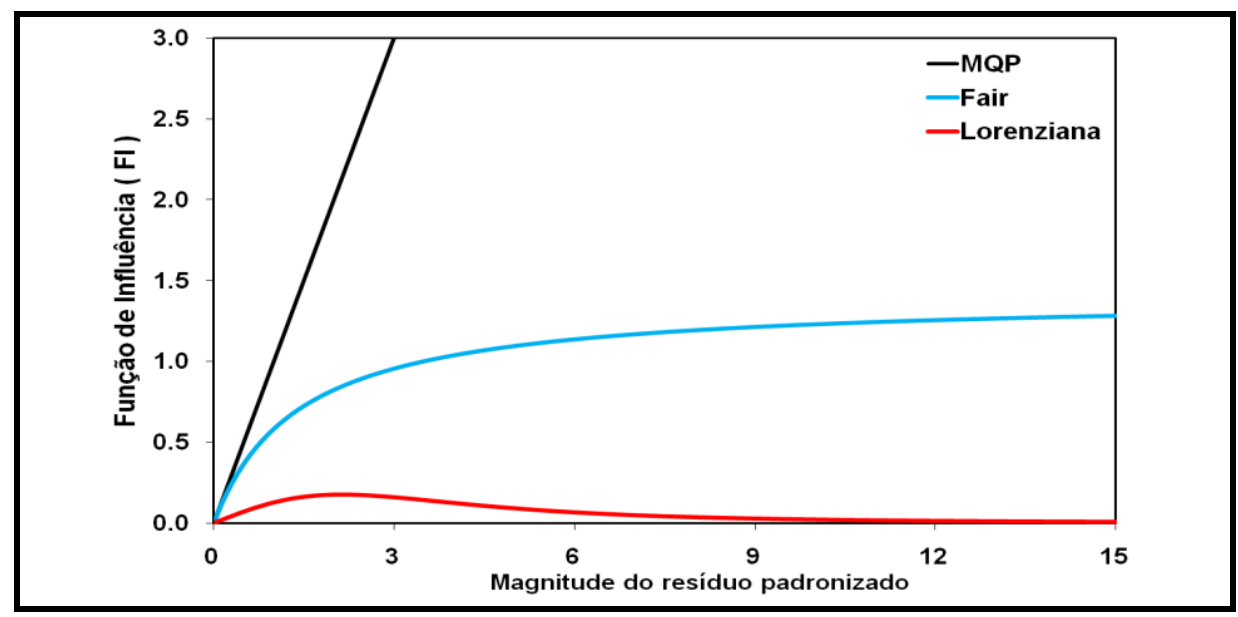

Figura 1 - Função de Influência (FI).

\section{O PROBLEMA DE PROCESSAMENTO DE MINÉRIO}

Este problema foi proposto originalmente por Alhaj-Dibo et al. (2008), sendo constituído por 16 correntes, cada uma das quais caracterizada pela vazão e por duas composições (que não necessariamente somam 1, pois deve existir mais componentes). Exceto pelas vazões das correntes: 1, 4 e 11, todas as demais variáveis são medidas. Desta forma o problema possui um total de 48 variáveis, sendo 3 não-medidas, todas observáveis. $\mathrm{O}$ fluxograma deste processo é ilustrado na Figura 1. O modelo matemático resultante, considerando estado estacionário, é apresentado na Equação 5. 


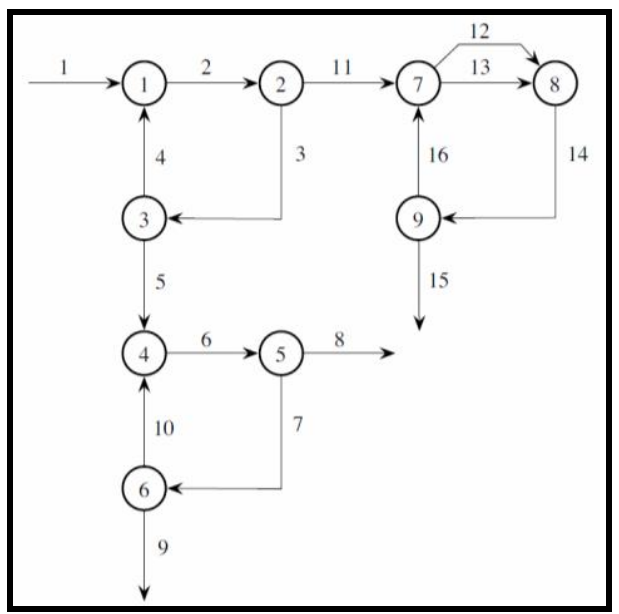

Figura 2 - Fluxograma de processamento de minério (Alhaj-Dibo et al. 2008).

$$
\begin{aligned}
& x_{1}+x_{4}-x_{2}=0 \\
& x_{2}-x_{3}-x_{11}=0 \\
& x_{3}-x_{4}-x_{5}=0 \\
& x_{5}+x_{10}-x_{6}=0 \\
& x_{6}-x_{7}-x_{8}=0 \\
& x_{7}-x_{9}-x_{10}=0 \\
& x_{11}+x_{16}-x_{12}-x_{13}=0 \\
& x_{12}+x_{13}-x_{14}=0 \\
& x_{14}-x_{15}-x_{16}=0 \\
& x_{1} \cdot y_{1,1}+x_{4} \cdot y_{1,4}-x_{2} \cdot y_{1,2}=0 \\
& x_{1} \cdot y_{2,1}+x_{4} \cdot y_{2,4}-x_{2} \cdot y_{2,2}=0 \\
& x_{2} \cdot y_{1,2}-x_{3} \cdot y_{1,3}-x_{11} \cdot y_{1,11}=0 \\
& x_{2} \cdot y_{2,2}-x_{3} \cdot y_{2,3}-x_{11} \cdot y_{2,11}=0 \\
& x_{3} \cdot y_{1,3}-x_{4} \cdot y_{1,4}-x_{5} \cdot y_{1,5}=0 \\
& x_{3} \cdot y_{2,3}-x_{4} \cdot y_{2,4}-x_{5} \cdot y_{2,5}=0 \\
& x_{5} \cdot y_{1,5}+x_{10} \cdot y_{1,10}-x_{6} \cdot y_{1,6}=0 \\
& x_{5} \cdot y_{2,5}+x_{10} \cdot y_{2,10}-x_{6} \cdot y_{2,6}=0 \\
& x_{6} \cdot y_{1,6}-x_{7} \cdot y_{1,7}-x_{8} \cdot y_{1,8}=0 \\
& x_{6} \cdot y_{2,6}-x_{7} \cdot y_{2,7}-x_{8} \cdot y_{2,8}=0 \\
& x_{7} \cdot y_{1,7}-x_{9} \cdot y_{1,9}-x_{10} \cdot y_{1,10}=0 \\
& x_{7} \cdot y_{2,7}-x_{9} \cdot y_{2,9}-x_{10} \cdot y_{2,10}=0 \\
& x_{11} \cdot y_{1,11}+x_{16} \cdot y_{1,16}-x_{12} \cdot y_{1,12}-x_{13} \cdot y_{1,13}=0 \\
& x_{11} \cdot y_{2,11}+x_{16} \cdot y_{2,16}-x_{12} \cdot y_{2,12}-x_{13} \cdot y_{2,13}=0 \\
& x_{12} \cdot y_{1,12}+x_{13} \cdot y_{1,13}-x_{14} \cdot y_{1,14}=0 \\
& x_{12} \cdot y_{2,12}+x_{13} \cdot y_{2,13}-x_{14} \cdot y_{2,14}=0 \\
& x_{14} \cdot y_{1,14}-x_{15} \cdot y_{1,15}-x_{16} \cdot y_{1,16}=0 \\
& x_{14} \cdot y_{2,14}-x_{15} \cdot y_{2,15}-x_{16} \cdot y_{2,16}=0 \\
&
\end{aligned}
$$


Alhaj-Dibo et al. (2008) consideraram o balanço de massa e o balanço de massa por componente. Neste modelo $\mathrm{x}_{\mathrm{i}}$ representa as vazões para as correntes (i) e $\mathrm{y}_{\mathrm{j}, \mathrm{i}}$ representa as composições dos componentes (j) nestas correntes.

\subsection{Critério de Avaliação}

Para avaliar a qualidade do resultado dos estimadores robustos sobre os dados corrompidos por erros grosseiros para as estimativas das variáveis medidas e não medidas será utilizado o critério SSE (Sum of Squared Error), apresentado na Equação 6.

$$
S S E=\sum\left(x_{\text {reconciliado }}-x_{\text {exato }}\right)^{2}+\sum\left(u_{\text {estimado }}-u_{\text {exato }}\right)^{2}
$$

Quanto menor for a SSE obtida, melhor terá sido o resultado, indicando uma diferença pequena entre os valores reconciliados /estimados e os exatos. Para a detecção do erro grosseiro utilizou-se o teste clássico para 95\% de confiança com base na distribuição Normal, apresentado na Equação 7, uma vez que se conhece $\sigma$.

$$
\left|x_{\text {reconcilialo }}-x_{\text {medido }}\right|>1.96 \sigma
$$

\section{RESULTADOS E DISCUSSÃO}

Na Tabela 1 são apresentados os resultados obtidos neste trabalho e por Alhaj-Dibo et al. (2008), bem como os valores medidos e respectivos desvios-padrão.

Tabela 1 - Resultados da RD para o problema de processamento de minério.

\begin{tabular}{ccccccccccccc}
\hline & \multicolumn{3}{c}{ Medidas } & \multicolumn{4}{c}{ Desvio-Padrão } & \multicolumn{3}{c}{ Alhaj-Dibo et al. (2008) } & \multicolumn{3}{c}{ EMSO (Lorenziana) } \\
\hline & $\mathrm{x}$ & $\mathrm{y}_{1}$ & $\mathrm{y}_{2}$ & $\mathrm{x}$ & $\mathrm{y}_{1}$ & $\mathrm{y}_{2}$ & $\mathrm{x}$ & $\mathrm{y}_{1}$ & $\mathrm{y}_{2}$ & $\mathrm{x}$ & $\mathrm{y}_{1}$ & $\mathrm{y}_{2}$ \\
\hline 1 & & $\mathbf{3 . 8 9}$ & 3.44 & & 0.389 & 0.344 & 22.580 & $\mathbf{2 . 4 3 0}$ & 3.460 & 22.7734 & $\mathbf{2 . 4 5 8 8}$ & 3.5044 \\
2 & 26.5 & 2.7 & 3.53 & 1.325 & 0.27 & 0.353 & 25.540 & 2.700 & 3.640 & 26.4172 & 2.7660 & 3.7025 \\
3 & $\mathbf{2 9 . 2}$ & 2.52 & 3.55 & 1.46 & 0.252 & 0.355 & $\mathbf{2 0 . 6 7 0}$ & 2.500 & 3.510 & $\mathbf{2 1 . 4 6 6 3}$ & 2.5807 & 3.5909 \\
4 & & 4.75 & 5.00 & & 0.475 & 0.5 & 2.960 & 4.760 & 4.980 & 3.6438 & 4.6859 & 4.9409 \\
5 & 18.32 & 2.09 & 3.29 & 0.916 & 0.209 & 0.329 & 17.710 & 2.120 & 3.270 & 17.8225 & 2.1503 & 3.3149 \\
6 & 22.02 & 2.46 & 3.51 & 1.101 & 0.246 & 0.351 & 21.870 & 2.500 & 3.510 & 21.9642 & 2.5151 & 3.5515 \\
7 & $\mathbf{2 0 . 8}$ & 2.9 & 3.74 & 1.04 & 0.29 & 0.374 & $\mathbf{1 2 . 2 9 0}$ & 2.900 & 3.720 & $\mathbf{1 2 . 3 1 8 6}$ & 2.9521 & 3.67370 \\
8 & 9.43 & 2.01 & $\mathbf{5 . 2}$ & 0.472 & 0.201 & 0.52 & 9.570 & 2.000 & $\mathbf{3 . 2 5 0}$ & 9.6458 & 1.9570 & $\mathbf{3 . 3 9 5 4}$ \\
9 & 8.01 & $\mathbf{3 . 7 1} *$ & 3.29 & 0.401 & 0.371 & 0.329 & 8.140 & $\mathbf{2 . 2 7 0}$ & 3.290 & 8.1768 & $\mathbf{2 . 3 7 8 4}$ & 3.2199 \\
10 & 4.14 & 4.15 & 4.55 & 0.207 & 0.415 & 0.455 & 4.150 & 4.140 & 4.560 & 4.1418 & 4.0848 & 4.5696 \\
11 & & 3.49 & 4.19 & & 0.349 & 0.419 & 4.870 & 3.550 & 4.160 & 4.9509 & 3.5694 & 4.1862 \\
12 & 6.56 & 3.63 & 4.34 & 0.328 & 0.363 & 0.434 & 6.650 & 3.500 & 4.280 & 6.5037 & 3.4677 & 4.2925 \\
13 & 1.04 & 8.71 & 6.32 & 0.052 & 0.871 & 0.632 & 1.040 & 8.590 & 6.300 & 1.0359 & 8.5671 & 6.3040 \\
14 & 7.38 & 4.24 & $\mathbf{6 . 6 5} *$ & 0.369 & 0.424 & 0.665 & 7.690 & 4.190 & $\mathbf{4 . 5 5 0}$ & 7.5396 & 4.1684 & $\mathbf{4 . 5 6 8 8}$ \\
15 & 4.99 & 3.49 & 4.1 & 0.25 & 0.349 & 0.41 & 4.870 & 3.550 & 4.160 & 4.9509 & 3.5694 & 4.1862 \\
16 & $\mathbf{7 . 6 9 *}$ & 5.15 & 5.18 & 0.385 & 0.515 & 0.518 & $\mathbf{2 . 8 2 0}$ & 5.290 & 5.230 & $\mathbf{2 . 5 8 8 6}$ & 5.3140 & 5.3006 \\
\hline
\end{tabular}

* Erros grosseiros. 
Alhaj-Dibo et al. (2008) utilizaram o estimador Normal Contaminada para minimizar/eliminar o efeito negativo dos erros grosseiros sobre as estimativas das variáveis. Neste trabalho utilizou-se o estimador Lorenziana, no pacote de Reconciliação do simulador EMSO e o otimizador IPOPT, para resolver o problema. Entretanto, para que o critério comparativo de desempenho SSE seja utilizado é necessário conhecer os valores "verdadeiros" (base de simulação) das variáveis. Alhaj-Dibo et al. (2008) disponibilizaram estes valores apenas para as variáveis corrompidas por erros grosseiros. Assim, apresentam-se na Tabela 2 os valores verdadeiros, reconciliados, medidos e seus respectivos desvios-padrão, bem como os critérios SSE e de DMEG, com base nas Equações 6 e 7, respectivamente.

Tabela 2 - Resultados da RD e DMEG para o problema de processamento de minério.

\begin{tabular}{ccccccccc}
\hline Variável & $\mathrm{x}_{3}$ & $\mathrm{x}_{7}$ & $\mathrm{x}_{16}$ & $\mathrm{y}_{1,1}$ & $\mathrm{y}_{1,9}$ & $\mathrm{y}_{2,8}$ & $\mathrm{y}_{2,14}$ & SSE \\
\hline Desvio-padrão & 1.46 & 1.04 & 0.385 & 0.389 & 0.371 & 0.52 & 0.665 & $*$ \\
Valor Verdadeiro & 21.2 & 12.8 & 2.69 & 2.39 & 2.21 & 3.2 & 4.65 & 0 \\
Valor Medido & 29.2 & 20.8 & 7.69 & 3.89 & 3.71 & 5.2 & 6.65 & 165.5 \\
Alhaj-Dibo et al. (2008) & 20.67 & 12.29 & 2.82 & 2.43 & 2.27 & 3.25 & 4.55 & 0.576 \\
EMSO (Lorenziana) & 21.4663 & 12.3186 & 2.58864 & 2.45881 & 2.37838 & 3.39543 & 4.56884 & $\mathbf{0 . 3 9 1}$ \\
DEG (Alhaj-Dibo) & 5.84 & 11.58 & 18.07 & 18.34 & 18.45 & 17.77 & 16.88 & $*$ \\
DEG EMSO & 5.30 & 11.56 & 18.23 & 18.32 & 18.37 & 17.67 & 16.87 & $*$ \\
\hline
\end{tabular}

$\mathrm{Na}$ Tabela 2 observa-se com clareza que o estimador Lorenziana (EMSO) obteve o menor valor para o critério SSE (0.391), e consequentemente o melhor resultado na presença de múltiplos erros grosseiros para o sistema de processamento de minério. Este resultado corrobora o anteriormente apresentado por Prata et al. (2008), que ressaltou o desempenho deste estimador e o de Welsch. Os estimadores Normal Contaminada e Lorenziana detectaram corretamente os erros grosseiros nas variáveis corrompidas, uma vez que todos os valores apresentados para o teste de detecção foram maiores do que 1.96. Estes resultados mostram, também, a essência da utilização dos estimadores robustos que são capazes de realizar o procedimento de RD e DMEG simultaneamente, evitando estratégias para DMEG com base em procedimentos iterativos, geralmente computacionalmente intensivos.

\section{CONCLUSÕES}

Este trabalho apresentou um estudo sobre o procedimento de RD e DMEG realizado simultaneamente por meio da utilização de estimadores robustos, em uma unidade de processamento de minério. Comparou-se o desempenho do estimador Normal Contaminada utilizado por Alhaj-Dibo et al. (2008) e o estimador Lorenziana, presente no pacote de reconciliação do simulador EMSO. O resultados mostraram melhor desempenho do estimador Lorenziana, o que corroborou o estudo prévio de Prata et al. (2008) que ressaltou o desempenho deste estimador e o de Welsch. Embora o estimador de Welsch não esteja presente no simulador EMSO é possível utilizá-lo, programando o problema de RD no pacote de otimização deste software. Isto será endereçado em outro trabalho. O desempenho do simulador EMSO pode ser comprovado em um exemplo não linear de dimensão considerada. 


\section{REFERÊNCIAS}

ALHAJ-DIBO, M.; MAQUIN, D.; RAGOT, J. Data reconciliation: A robust approach using a contaminated distribution. Control Eng. Prac., v. 16, p. 159-170, 2008.

BAZIN, C.; HODOUIN, D.; DUCHESNE, C.; THIBAULT, J.; TRUSIAK, A. R. Reconciliation of mass and energy data measurements: application to a rotary dryer. Can. Metall. Quart., v. 37, p. 333-342, 1998.

BAZIN, C.; ROCHON-TEMBLAY, S.; GOSSELIN, C. Estimation of gas flow rates and pellets temperature in an iron oxide induration furnace. Can. Metall. Quart., v. 42, p. 301-312, 2003.

De ANDRADE LIMA, L. R. P. Nonlinear data reconciliation in gold processing plants. Miner. Eng., v. 19, p. 938-951.

DU, Y.G.; THIBAULT; J., HODOUIN, D., Data reconciliation for simulated flotation process. Artif. Intell. Eng., v. 11, pp. 357-364, 1997.

EKSTEEN, J. J; FRANK, S. J., REUTER, M. A. Dynamic structures in variance based data reconciliation adjustments for a chromite smelting furnace. Miner. Eng., v. 15, p. 931943, 2002.

HODOUIN, D.; GARON, M.; RÉMILLARD, M.; THÉRIEN, M. Assessment of precious metal distribution in Lac Mattagami flotation plant by computer mass balance calculation. Can. Inst. Min. Metal. Bull., v.81, p. 62-69, 1988.

HODOUIN, D.; GELPE, T.; EVERELL, M. D. Sensitivity analysis of material balance calculations- an application to a cement clinker grinding circuit. Powder Technol., v. 3, p.139-153, 1982.

HODOUIN, D.; KASONGO, T.; KOUAMÉ, E.; EVERELL, M. D. BILMAT: an algorithm for material balancing mineral processing circuits: applications to comminution, desliming and flotation units. Can. Inst. Min. Metal. Bull.,v., 74 p.123-131, 1981.

MULAR, A. L.; BRADBURN, R.G.; FLINTOFF, B. C.; LARSEN, C. R. Mass balance of a grinding circuit. Can. Inst. Min. Metal. Bull., v.69, p. 124-129, 1976.

PRATA, D. M.; PINTO, J. C.; LIMA, E. L. Comparative analysis of robust estimators on nonlinear dynamic data reconciliation. Comput. Aided Chem. Eng., v. 25, p. 501-506, 2008.

SBARBARO, D.; DEL VILLAR, R. Advanced Control and Supervision of Mineral Processing Plants. London: Editora Spriger, 2010.

SOARES, R. P.; SECCHI, A. R. EMSO: Environment for Modelling, Simulation and Optimisation. Comput. Aided Chem. Eng., v. 14, p. 947-952, 2003.

TJOA, I. B.; BIEGLER, L. T. Simultaneous strategy for data reconciliation and gross error detection of nonlinear systems. Comput. Chem. Engng., v 15, p. 679-690, 1991.

WHITE, J. W.; WINSLOW, R. L.; ROSSITER, G. J. A useful technique for metallurgical mass balances - applications in grinding. Int. J. Miner. Proc., v. 4, p. 39-49, 1977.

WIEGEL, R. I. Advances in mineral processing material balance. Canad. Metall. Q., v. 11, p. 413-424, 1972. 